Bulletin of the Section of Logic

Volume 50/1 (2021), pp. 35-53

https://doi.org/10.18778/0138-0680.2020.24

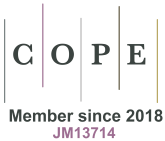

Natalya Tomova*

\title{
A SEMI-LATTICE OF FOUR-VALUED LITERAL-PARACONSISTENT-PARACOMPLETE LOGICS
}

\begin{abstract}
In this paper, we consider the class of four-valued literal-paraconsistent-paracomplete logics constructed by combination of isomorphs of classical logic $C P C$. These logics form a 10-element upper semi-lattice with respect to the functional embeddinig one logic into another. The mechanism of variation of paraconsistency and paracompleteness properties in logics is demonstrated on the example of two four-element lattices included in the upper semi-lattice. Functional properties and sets of tautologies of corresponding literal-paraconsistent-paracomplete matrices are investigated. Among the considered matrices there are the matrix of Puga and da Costa's logic $V$ and the matrix of paranormal logic $P^{1} I^{1}$, which is the part of a sequence of paranormal matrices proposed by V. Fernández.

Keywords: Four-valued logics, paraconsistent logics, paracomplete logics, isomorphisms, literal-paraconsistent-paracomplete logics, semi-lattice of logics.

\section{Introduction}

Literal-paraconsistent-paracomplete logics (or LPP logics) are logics in which paraconsistency and/or paracompleteness occurs only at the level of literals, that is, formulas that are propositional letters or their iterated negations [13, p. 478].

${ }^{*}$ I want to thank the referee for the helpful comments on an earlier draft of this paper.
\end{abstract}

Presented by: Janusz Ciuciura

Received: May 22, 2020

Published online: November 13, 2020

(C) Copyright for this edition by Uniwersytet Łódzki, Łódź 2020 
The class of LPP logics is well studied. Among the LPP logics considered in this paper, there are the logics described in [19], [18], [21], [16], [7], [12], [5], [15].

There are several algorithms of constructing classes of such logics, for example, we can note the following ones: (1) construction of LPP logics by combination of isomorphs of classical logic $C P C$ [11]; (2) construction of LPP logics by using literal-paraconsistent-paracomplete matrices (or LPPmatrices) [13]. But the classes of LPP logics constructed by these two methods are not equal: the class obtained by (1) is a subclass of the class obtained by (2).

It is known that Sette's three-valued paraconsistent logic $P^{1}$ [19] and three-valued paracomplete logic $I^{1}[21]$ can be represented as combinations of two three-valued isomorphs of classical propositional logic, contained in three-valued Bochvar's logic $B_{3}$ [11].

The paper [23] briefly describes the result of the application of the first method of constructing LPP logics to the four-valued case. So, the sixteen LPP logics form the upper semi-lattice with respect to the functional inclusion.

This paper is devoted to the study of four-valued propositional LPP logics that form the above-mentioned upper semi-lattice. Some properties of the class of four-valued paranormal logics constituting the supremum of the said semi-lattice were regarded in paper [22]. In this paper, we consider two interesting lattices of LPP logics that are included in the upper semilattice.

As a result, it allows us to demonstrate some properties of the negation operation in LPP logics, to compare LPP logics by functional properties and classes of tautologies.

The paper is stuctured as follows.

In the next section, we introduce some basic definitions. In the third section, we present the upper semi-lattice of four-valued LPP logics. In the next, we select two four-element lattices of LPP logics included in that semi-lattice, and consequently consider the properties of the logics that constitute these lattices. 


\section{Basic definitions}

There are several approaches to the representation and analysis of logical systems. In this paper, logical systems are represented by means of logical matrices. Let us introduce some basic definitions.

Let $\mathcal{L}$ be a sentential language, i.e. $\mathcal{L}=\left\langle F\right.$ or $\left., F_{1}, \ldots, F_{m}\right\rangle$ is an algebra generated by a set of variables $\operatorname{Var}=\{p, q, r \ldots\}$. Elements of For are generated from variables with the use of operations $F_{1}, \ldots, F_{m}$, representing sentential conectives.

Let $\mathcal{A}=\left\langle V, f_{1}, \ldots, f_{m}\right\rangle$ be an algebra similar to $\mathcal{L}$, where $V$ is the set of truth-values and each $f_{i}$ is a function on $V$ with the same arity as $F_{i}$.

Definition 2.1. A structure $\mathfrak{M}=\langle\mathcal{A}, D\rangle$ with $\mathcal{A}$ being an algebra similar to a propositional language $\mathcal{L}$ and $D \subseteq V$ - a non-empty subset of the universe of $\mathcal{A}$ is called a logical matrix for $\mathcal{L}$. Elements of $D$ are called designated elements of $\mathfrak{M}$.

Throughout the paper we use the same symbols both for the propositional connective and the corresponding function on $V$.

Definition 2.2. A valuation $v$ of the formula $A$ in the matrix $\mathfrak{M}$ for the language $\mathcal{L}$ is a homomorphism from $\mathcal{L}$ into $\mathcal{A}=\left\langle V, f_{1}, \ldots, f_{m}\right\rangle$, such that

1. if $p$ is a propositional variable, then $v(p) \in V$;

2. if $A_{1}, A_{2}, \ldots, A_{n}$ are formulas and $F^{n}$ is an $n$-ary connective of language $\mathcal{L}$, then $v\left(F^{n}\left(A_{1}, A_{2}, \ldots, A_{n}\right)\right)=f^{n}\left(v\left(A_{1}\right), v\left(A_{2}\right), \cdots, v\left(A_{n}\right)\right)$, where $f^{n}$ is a function on $V$ corresponding to $F^{n}$.

Definition 2.3. Some formula $A$ is a tautology in $\mathfrak{M}$ (abbreviated to $\vDash_{\mathfrak{M}} A$ ), iff for every valuation $v$ in $\mathfrak{M}$ it is true that $v(A) \in D$.

Definition 2.4. The theory generated by $\mathfrak{M}$ is the set of all tautologies in $\mathfrak{M}$. It is denoted by $E(\mathfrak{M})$.

Definition 2.5. The formula $B$ logically follows from the set of formulas $\Gamma=\left\{A_{1}, A_{2}, \ldots, A_{n}\right\}$ in $\mathfrak{M}$ (abbreviated to $\Gamma \vDash_{\mathfrak{M}} B$ ), iff there is no such valuation $v$ in $\mathfrak{M}$, such that $v\left(A_{i}\right) \in D$ for each $A_{i} \in \Gamma$ and $v(B) \notin D$.

Definition 2.6. The consequence relation generated by $\mathfrak{M}$ is the set $C n(\mathfrak{M})$ of ordered pairs $\langle\Gamma, B\rangle$, such that for every valuation $v$ in $\mathfrak{M}$ if $v(\Gamma) \subseteq D$, then $v(B) \in D$. 
Let $L_{1}$ be a logic represented by matrix $\mathfrak{M}_{1}$ with the set of function $F_{1}$ and $L_{2}$ a logic represented by matrix $\mathfrak{M}_{2}$ with the set of functions $F_{2}$.

Definition 2.7. A logic $L_{1}$ is functionally included in a logic $L_{2}$ iff every function of $F_{1}$ can be expressed by a superposition of functions of $F_{2}$.

Definition 2.8. A logic $L_{1}$ is functionally equivalent to a logic $L_{2}$ iff

(1) $L_{1}$ is functionally included in $L_{2}$ and

(2) $L_{2}$ is functionally included in $L_{1}$.

Definition 2.9. A logic $L_{1}$ is a fragment of a logic $L_{2}$ iff $L_{1}$ is functionally included in $L_{2}$, but $L_{2}$ is not functionally included in $L_{1}$, i.e., the opposite does not hold.

Definition 2.10. Some fragment of a logic $L$ is said to be an isomorph of classical propositional logic iff $L$ has the classical set of tautologies and the classical consequence relation.

Different formal criteria may be used for the construction of paralogics. Jaśkowski's criteria for constructing paraconsistent logic is considered in some detail in [10]. In our investigation we use its "implicative-negative" part:

Definition 2.11. In a system of paraconsistent logic, the Duns Scotus law $A \supset(\neg A \supset B)^{1}$ is not valid, for some formulas A, B.

Definition 2.12. In a paracomplete logic system, the Clavius law $(\neg A \supset$ A) $\supset A$ ) is not valid, for some formula $\mathrm{A}$ (see [4]).

DeFinition 2.13. Logics, which are simultaneously paraconsistent and paracomplete, are called paranormal logics.

If logical systems are represented as theories (as classes of tautologies), this criteria best fits the scope.

In terms of logical consequence, logic is paraconsistent, iff its consequence relation is not explosive (principle of explosion: $A, \neg A \vDash B$, see [17]). The logic is paracomplete, iff there is a set of formulas $\Gamma$ and formulas $A$ and $B$, such that $\Gamma, A \vDash B$ and $\Gamma, \neg A \vDash B$, but $\Gamma \not \models B$ (see [1, p. 1092]).

\footnotetext{
${ }^{1}$ The implicational law of over-completeness in Jaśkowski's notation (see [8]).
} 


\section{An upper semi-lattice of LPP logics}

In the book [12, pp. 56-79] a class of four-valued LPP logics obtained by combining isomorphs of classical logic $C P C$ is presented. These four fourvalued $C P C$ isomorphs are the fragments of Bochvar's four-valued logic $B_{4}$ [2, p. 289], which is determined by the matrix

$$
\mathfrak{M}_{4}^{B}=\left\langle\{0,1 / 3,2 / 3,1\}, \sim, \cap, \cup, J_{0}, J_{1 / 3}, J_{2 / 3}, J_{1},\{1\}\right\rangle,
$$

where $\sim x=1-x$, and $J$-operators, $\cap$ and $\cup$ are defined by the following truth-tables (cf. [2, p. 294]):

\begin{tabular}{|c|c|c|c|c|}
\hline$x$ & $J_{0}(x)$ & $J_{1 / 3}(x)$ & $J_{2 / 3}(x)$ & $J_{1}(x)$ \\
\hline 1 & 0 & 0 & 0 & 1 \\
$2 / 3$ & 0 & 0 & 1 & 0 \\
$1 / 3$ & 0 & 1 & 0 & 0 \\
0 & 1 & 0 & 0 & 0 \\
\hline
\end{tabular}

\begin{tabular}{|c|cccc|}
\hline$\cap$ & 1 & $2 / 3$ & $1 / 3$ & 0 \\
\hline 1 & 1 & $2 / 3$ & $1 / 3$ & 0 \\
$2 / 3$ & $2 / 3$ & $2 / 3$ & $1 / 3$ & $1 / 3$ \\
$1 / 3$ & $1 / 3$ & $1 / 3$ & $1 / 3$ & $1 / 3$ \\
0 & 0 & $1 / 3$ & $1 / 3$ & 0 \\
\hline
\end{tabular}

\begin{tabular}{|c|cccc|}
\hline$\cup$ & 1 & $2 / 3$ & $1 / 3$ & 0 \\
\hline 1 & 1 & $2 / 3$ & $2 / 3$ & 1 \\
$2 / 3$ & $2 / 3$ & $2 / 3$ & $2 / 3$ & $2 / 3$ \\
$1 / 3$ & $2 / 3$ & $2 / 3$ & $1 / 3$ & $1 / 3$ \\
0 & 1 & $2 / 3$ & $1 / 3$ & 0 \\
\hline
\end{tabular}

Functional properties of Bochvar's logic $B_{3}$ are determined by the union of two types of connectives - internal and external ${ }^{2}$. In the three-valued case internal connectives can be translated into external ones in two different ways [9, pp. 212-213]. These two translations provide construction of two fragments of $B_{3}$ isomorphic with $C P C$. In the one isomorph the truth-value $1 / 2$ is identified with 0 and in the other - with 1 .

In the four-valued case there are four translation functions: $f_{1}(x), f_{2}(x)$, $f_{3}(x)$ and $f_{4}(x)$. They have the following properties:

${ }^{2}$ A function $f$ on $V$ into $V$ with arity $n$ is called external iff for any values $x_{1} \ldots x_{n}$ we have either $f\left(x_{1}, \ldots, x_{n}\right)=0$ or $f\left(x_{1}, \ldots, x_{n}\right)=1$. 
(1) $f_{1}(x)$ is $J_{1}(x)$ and takes the truth-values $2 / 3$ and $1 / 3$ to 0 ;

(2) $f_{2}(x)$ is $\sim J_{0}(x)$ and takes $2 / 3$ and $1 / 3$ to 1 ;

(3) $f_{3}(x)$ is $J_{1}(x) \cup J_{2 / 3}(x)$ and takes $2 / 3$ to 1 and $1 / 3$ to 0 ;

(4) $f_{4}(x)$ is $J_{1}(x) \cup J_{1 / 3}(x)$ and takes $2 / 3$ to 0 and $1 / 3$ to 1 .

\begin{tabular}{|c|c|c|c|c|}
\hline$x$ & $f_{1}(x)$ & $f_{2}(x)$ & $f_{3}(x)$ & $f_{4}(x)$ \\
\hline 1 & 1 & 1 & 1 & 1 \\
$2 / 3$ & 0 & 1 & 1 & 0 \\
$1 / 3$ & 0 & 1 & 0 & 1 \\
0 & 0 & 0 & 0 & 0 \\
\hline
\end{tabular}

Using $f_{1}(x), f_{2}(x), f_{3}(x)$ and $f_{4}(x)$ analogously as it was done for $B_{3}$, we can construct four external negations and four external implications:

$$
\neg_{i} x:=\sim f_{i}(x) \text { and } x \rightarrow_{i} y:=\neg_{i} x \cup f_{i}(y)(i \in\{1,2,3,4\}),
$$

which are defined by the following truth-tables:

\begin{tabular}{|c|c|c|c|c|}
\hline$x$ & $\neg_{1} x$ & $\neg_{2} x$ & $\neg_{3} x$ & $\neg_{4} x$ \\
\hline 1 & 0 & 0 & 0 & 0 \\
$2 / 3$ & 1 & 0 & 0 & 1 \\
$1 / 3$ & 1 & 0 & 1 & 0 \\
0 & 1 & 1 & 1 & 1 \\
\hline
\end{tabular}

\begin{tabular}{|c|cccc|}
\hline$\rightarrow_{1}$ & 1 & $2 / 3$ & $1 / 3$ & 0 \\
\hline 1 & 1 & 0 & 0 & 0 \\
$2 / 3$ & 1 & 1 & 1 & 1 \\
$1 / 3$ & 1 & 1 & 1 & 1 \\
0 & 1 & 1 & 1 & 1 \\
\hline
\end{tabular}

\begin{tabular}{|c|cccc|}
\hline$\rightarrow_{2}$ & 1 & $2 / 3$ & $1 / 3$ & 0 \\
\hline 1 & 1 & 1 & 1 & 0 \\
$2 / 3$ & 1 & 1 & 1 & 0 \\
$1 / 3$ & 1 & 1 & 1 & 0 \\
0 & 1 & 1 & 1 & 1 \\
\hline
\end{tabular}




\begin{tabular}{|c|cccc|}
\hline$\rightarrow_{3}$ & 1 & $2 / 3$ & $1 / 3$ & 0 \\
\hline 1 & 1 & 1 & 0 & 0 \\
$2 / 3$ & 1 & 1 & 0 & 0 \\
$1 / 3$ & 1 & 1 & 1 & 1 \\
0 & 1 & 1 & 1 & 1 \\
\hline
\end{tabular}

\begin{tabular}{|c|cccc|}
\hline$\rightarrow_{4}$ & 1 & $2 / 3$ & $1 / 3$ & 0 \\
\hline 1 & 1 & 0 & 1 & 0 \\
$2 / 3$ & 1 & 1 & 1 & 1 \\
$1 / 3$ & 1 & 0 & 1 & 0 \\
0 & 1 & 1 & 1 & 1 \\
\hline
\end{tabular}

Four-valued $C P C$ isomorphs are determined by the following matrices:

$$
\begin{aligned}
& \mathfrak{M}_{1}=\left\langle\{0,1 / 3,2 / 3,1\}, \neg_{1}, \rightarrow_{1},\{1\}\right\rangle, \\
& \mathfrak{M}_{2}=\left\langle\{0,1 / 3,2 / 3,1\}, \neg_{2}, \rightarrow_{2},\{1,2 / 3,1 / 3\}\right\rangle, \\
& \mathfrak{M}_{3}=\left\langle\{0,1 / 3,2 / 3,1\}, \neg_{3}, \rightarrow_{3},\{1,2 / 3\}\right\rangle, \\
& \mathfrak{M}_{4}=\left\langle\{0,1 / 3,2 / 3,1\}, \neg_{4}, \rightarrow_{4},\{1,1 / 3\}\right\rangle .
\end{aligned}
$$

Combining the operations $\neg_{i}, \rightarrow_{j}(i, j \in\{1,2,3,4\})$ of the isomorphs we construct the class of four-valued literal LPP logics. Let us present the corresponding matrices:

$$
\begin{aligned}
& \text { paraconsistent } \\
& \mathfrak{M}_{5}=\left\langle\{0,1 / 3,2 / 3,1\}, \neg_{1}, \rightarrow_{2},\{1,2 / 3,1 / 3\}\right\rangle, \\
& \mathfrak{M}_{6}=\left\langle\{0,1 / 3,2 / 3,1\}, \neg_{3}, \rightarrow_{2},\{1,2 / 3,1 / 3\}\right\rangle, \\
& \mathfrak{M}_{7}=\left\langle\{0,1 / 3,2 / 3,1\}, \neg_{4}, \rightarrow_{2},\{1,2 / 3,1 / 3\}\right\rangle, \\
& \mathfrak{M}_{8}=\left\langle\{0,1 / 3,2 / 3,1\}, \neg_{1}, \rightarrow_{3},\{1,2 / 3\}\right\rangle, \\
& \mathfrak{M}_{9}=\left\langle\{0,1 / 3,2 / 3,1\}, \neg_{1}, \rightarrow_{4},\{1,1 / 3\}\right\rangle .
\end{aligned}
$$

\section{paracomplete}

$$
\begin{aligned}
& \mathfrak{M}_{10}=\left\langle\{0,1 / 3,2 / 3,1\}, \neg_{2}, \rightarrow_{1},\{1\}\right\rangle, \\
& \mathfrak{M}_{11}=\left\langle\{0,1 / 3,2 / 3,1\}, \neg_{3}, \rightarrow_{1},\{1\}\right\rangle, \\
& \mathfrak{M}_{12}=\left\langle\{0,1 / 3,2 / 3,1\}, \neg_{4}, \rightarrow_{1},\{1\}\right\rangle, \\
& \mathfrak{M}_{13}=\left\langle\{0,1 / 3,2 / 3,1\}, \neg_{2}, \rightarrow_{3},\{1,2 / 3\}\right\rangle, \\
& \mathfrak{M}_{14}=\left\langle\{0,1 / 3,2 / 3,1\}, \neg_{2}, \rightarrow_{4},\{1,1 / 3\}\right\rangle .
\end{aligned}
$$




$$
\begin{aligned}
& \text { paranormal } \\
& \mathfrak{M}_{15}=\left\langle\{0,1 / 3,2 / 3,1\}, \neg_{4}, \rightarrow_{3},\{1,2 / 3\}\right\rangle, \\
& \mathfrak{M}_{16}=\left\langle\{0,1 / 3,2 / 3,1\}, \neg_{3}, \rightarrow_{4},\{1,1 / 3\}\right\rangle .
\end{aligned}
$$

As a result, a ten-element upper semi-lattice (see Figure 1) is constructed with respect to the functional embedding of matrices that define literal LPP logics and the isomorphs themselves ${ }^{3}$.

The question about the functional inclusion one LPP logic to another was solved by A. Nepeivoda (see [23]).

The resulting semi-lattice allows us to build visualization for constructing LPP logics by the combination of $C P C$ isomorphs. Note that the isomorphs themselves are included in our class of LPP logics as a degenerate case. The four isomorphs differ by functional properties and have the least expressive power.

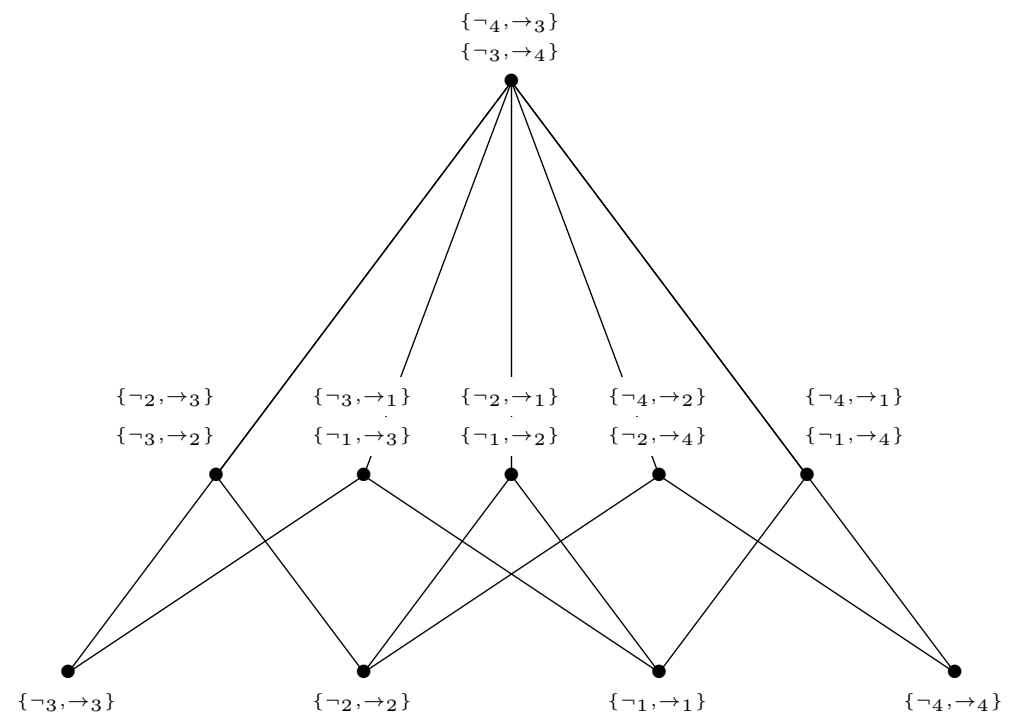

Figure 1. An upper semi-lattice

${ }^{3}$ The sets of basic operations of the corresponding logical matrices are indicated as semilattice elements. 
The above structure is indeed an upper semi-lattice, since there is a supremum for any pair of its elements. In some cases, this is clearly seen in the construction of upper semi-lattices, in other cases it requires proof. Let us give the corresponding proof. To do this, it is sufficient to prove the following proposition:

Proposition 3.1. The operations of the set $\left\{\rightarrow_{3}, \neg_{4}\right\}$ are definable by the sets of operations:

(1) $\left\{\neg_{2}, \rightarrow_{3}\right\}$ and $\left\{\neg_{3}, \rightarrow_{1}\right\}$;

(2) $\left\{\neg_{3}, \rightarrow_{1}\right\}$ and $\left\{\neg_{2}, \rightarrow_{1}\right\}$;

(3) $\left\{\neg_{2}, \rightarrow_{1}\right\}$ and $\left\{\neg_{4}, \rightarrow_{2}\right\}$;

(4) $\left\{\neg_{4}, \rightarrow_{2}\right\}$ and $\left\{\neg_{4}, \rightarrow_{1}\right\}$.

Proof: For (1), it is sufficient to define $\neg_{4}$ by the sets of functions $\left\{\neg_{2}, \rightarrow_{3}\right\}$ and $\left\{\neg_{3}, \rightarrow_{1}\right\}$. The function $\wedge_{3}$ can be defined by $\left\{\neg_{2}, \rightarrow_{3}\right\}$ the following way:

$$
x \wedge_{3} y:=\neg_{2}\left(x \rightarrow_{3} \neg_{2} y\right) .
$$

Further, since the sets $\left\{\neg_{3}, \rightarrow_{1}\right\}$ and $\left\{\neg_{2}, \rightarrow_{3}\right\}$ are functionally equivalent (Fact 1), we have:

$$
\neg_{4} x:=\left(\neg_{3} x \rightarrow_{3} \neg_{2} x\right) \wedge_{3}\left(x \rightarrow_{3} \neg_{1} x\right) .
$$

For (2), it is sufficient to define $\neg_{4}$ and $\rightarrow_{3}$ by the sets of functions $\left\{\neg_{3}, \rightarrow_{1}\right\}$ and $\left\{\neg_{2}, \rightarrow_{1}\right\}$. Due to Fact 1 , it is obvious that the function $\rightarrow_{3}$ is definable. The function $\neg_{4}$ could be defined in the same way as it was done in the proof of (1).

For (3), it is sufficient to define $\neg_{3}$ and $\rightarrow_{4}$ by the sets of functions $\left\{\neg_{2}, \rightarrow_{1}\right\}$ and $\left\{\neg_{4}, \rightarrow_{2}\right\}$. Since the sets $\left\{\neg_{4}, \rightarrow_{2}\right\}$ and $\left\{\neg_{2}, \rightarrow_{4}\right\}$ are functionally equivalent (Fact 2), it is obvious that the function $\rightarrow_{4}$ is definable. The function $\neg_{3}$ could be defined in the following way. Since the function $\wedge_{1}$ is defined by $\left\{\neg_{2}, \rightarrow_{1}\right\}$ :

$$
x \wedge_{1} y:=\neg_{2}\left(x \rightarrow_{1} \neg_{2} y\right),
$$

and the sets of functions $\left\{\neg_{2}, \rightarrow_{1}\right\}$ and $\left\{\neg_{1}, \rightarrow_{2}\right\}$ are functionally equivalent, we have:

$$
\neg_{3} x:=\left(\neg_{4} x \rightarrow_{1} \neg_{2} x\right) \wedge_{1} \neg_{1} x .
$$

For (4), it is sufficient to define $\neg_{3}$ and $\rightarrow_{4}$ by the sets of functions $\left\{\neg_{4}, \rightarrow_{2}\right\}$ and $\left\{\neg_{4}, \rightarrow_{1}\right\}$. Due to Fact 2 , it is obvious that the function $\rightarrow_{4}$ is definable. Since the function $\wedge_{2}$ is defined by $\left\{\neg_{4}, \rightarrow_{2}\right\}$ :

$$
x \wedge_{2} y:=\neg_{4}\left(x \rightarrow_{2} \neg_{4} y\right),
$$


and the sets of functions $\left\{\neg_{4}, \rightarrow_{1}\right\}$ and $\left\{\neg_{1}, \rightarrow_{4}\right\}$ are functionally equivalent, and that Fact 2 takes place, we have:

$\neg_{3} x:=\left(\neg_{4} x \rightarrow_{2} \neg_{2} x\right) \wedge_{2} \neg_{1} x$.

In paper [9] we consruct a four-element lattice of three-valued literal LPP logics with respect to the possesion of paraconsistency and paracompleteness properties. And the theorem on the functional equivalence of the LPP logics that form this lattice was proved (p. 230).

The four-valued case is more complicated. The upper semi-lattice of four-valued litearal LPP logics contains a number of four-element lattices with respect to the functional embeddinig one logic into another, on one hand, and possesion of paraconsistency and paracompleteness properties, on the other. In the next section, we consider two interesting lattices.

\section{Four-element lattices of four-valued LPP logics}

Let us consider the matrices: $\mathfrak{M}_{3}, \mathfrak{M}_{6}, \mathfrak{M}_{8}, \mathfrak{M}_{11}, \mathfrak{M}_{13}, \mathfrak{M}_{15}, \mathfrak{M}_{16}$. The LPP logics determined by the foregoing matrices form two four-element lattices (see Fugure 2 and Fugure 3 ) with respect to the possesion of paraconsistency and paracompleteness properties, on one hand, and with respect to the functional embedding of logics (corresponding classes of matrix's operations), on the other.

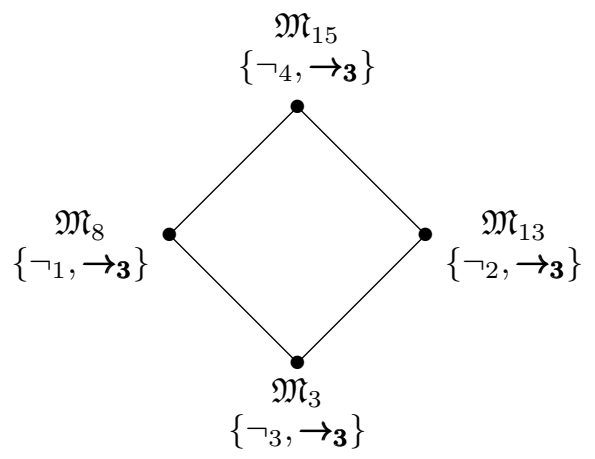

Figure 2. Lattice 1 


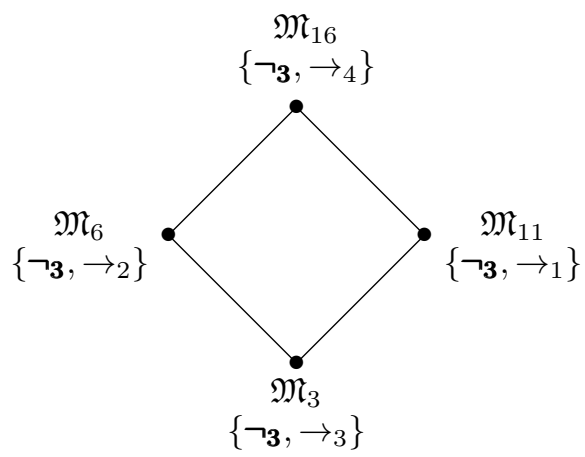

Figure 3. Lattice 2

Lattices in Figure 2 and Figure 3 are included in the upper semi-lattice in Figure 1.

Let us consider the properties of the logics that constitute these lattices.

(1) Logics introduced by the matrices $\mathfrak{M}_{6}$ and $\mathfrak{M}_{8}$ are paraconsistent.

(2) Logics introduced by the matrices $\mathfrak{M}_{11}$ and $\mathfrak{M}_{13}$ are paracomplete.

(3) Logics introduced by the matrices $\mathfrak{M}_{15}$ and $\mathfrak{M}_{16}$ are paranormal.

\subsection{Functional properties}

By the construction of the upper semi-lattice (see Fugure 1), it is obvious that paraconsistent logics with operations $\left\{\neg_{3}, \rightarrow_{2}\right\}$ and $\left\{\neg_{1}, \rightarrow_{3}\right\}$ (matrices $\mathfrak{M}_{6}$ and $\left.\mathfrak{M}_{8}\right)$ are different in functional properties. Similarly that about paracomplete logics with operations $\left\{\neg_{3}, \rightarrow_{1}\right\}$ and $\left\{\neg_{2}, \rightarrow_{3}\right\}$ (matrices $\mathfrak{M}_{11}$ and $\left.\mathfrak{M}_{13}\right)$. But the matrices $\mathfrak{M}_{6}$ and $\mathfrak{M}_{13}$ are functionally equivalent, and the same situation takes place for matrices $\mathfrak{M}_{8}$ and $\mathfrak{M}_{11}$. Notice that we have a similar property for three-valued logics: the matrices corresponding to paraconsistent logic $P^{1}[19]$ and paracomplete logic $I^{1}$ [21] are also functionally equivalent $[12$, p. 222].

Paranormal logics with operations $\left\{\neg_{4}, \rightarrow_{3}\right\}$ and $\left\{\neg_{3}, \rightarrow_{4}\right\}$ (matrices $\mathfrak{M}_{15}$ and $\left.\mathfrak{M}_{16}\right)$ are functionally equivalent. In paper [22, p. 81-82] it is proved that these logical matrices correspond to the class of all external four-valued functions. 
Also, there are well-known four-valued logics that are functionally equivalent to $\mathfrak{M}_{15}\left(\mathfrak{M}_{16}\right)$. Logic $I^{1} P^{1}$, which is the part of a hierarchy of paranormal logics called $I^{n} P^{k}$, introduced by V. Fernández [6]. A sound and complete axiomatization for each $I^{n} P^{k}$ using the techniques of RosserTurquette was defined in [6]. In [5, p. 88] L. Devyatkin construct the four-valued matrices for the $\operatorname{logics} P^{1}$ and $I^{1}$. And he shows that the matrix of $I^{1} P^{1}$ constitutes a functional extension of $P^{1}$ and $I^{1}$ and this entails that $I^{1} P^{1}$ is a linguistic variant of a common linguistic extension of $P^{1}$ and $I^{1}$.

The matrix of $I^{1} P^{1}$ coincides with the matrix $\mathfrak{M}_{15}$.

Logic $V$ (see [18, p. 208] for the corresponding matrix) was introdused by L.Z. Puga and N.C.A. da Costa after ideas on the "imaginary logic" by N.A. Vasiliev. Corresponding matrix is functionally equivalent to the matrix $\mathfrak{M}_{15}\left(\mathfrak{M}_{16}\right)$.

In $\left[16\right.$, p. 89] V.M. Popov introduced the matrix $\mathfrak{M}_{0}$ (this matrix coincides with the matrix of logic $V)$, where truth-tables for $\neg$ and $\rightarrow(\neg 4$ and $\rightarrow_{3}$ in our notation), may be viewed as four-valued generalizations of $P^{1}$ 's and $I^{1}$ 's tables.

\subsection{Classes of tautologies}

In this section, we analyze the theories (sets of tautologies) generated by the foregoing matrices.

Paraconsistent logic $P^{1}$ [19] and paracomplete logic $I^{1}$ [21] play a significant role in our analysis. The calculi $P^{1}$ and $I^{1}$ are expressed in a language using negation and implication as a primitives.

$P^{1}$ is axiomatized by the following axiom schemata:

(A1) $A \rightarrow(B \rightarrow A)$

(A2) $(A \rightarrow(B \rightarrow C)) \rightarrow((A \rightarrow B) \rightarrow(A \rightarrow C))$

(A3) $(\neg A \rightarrow \neg B) \rightarrow((\neg A \rightarrow \neg \neg B) \rightarrow A)$

(A4) $(A \rightarrow B) \rightarrow \neg \neg(A \rightarrow B)$

Inference rule: modus ponens [20].

The matrix $\mathfrak{M}^{P 1}=\left\langle\{1,1 / 2,0\}, \neg P 1, \rightarrow_{P 1},\{1,1 / 2\}\right\rangle$, where $\neg P 1$ and $\rightarrow_{P 1}$ are defined by the tables 


\begin{tabular}{|c|c|}
\hline$x$ & $\neg P 1$ \\
\hline 1 & 0 \\
$1 / 2$ & 1 \\
0 & 1 \\
\hline
\end{tabular}

\begin{tabular}{|c|ccc|}
\hline$\rightarrow_{P 1}$ & 1 & $1 / 2$ & 0 \\
\hline 1 & 1 & 1 & 0 \\
$1 / 2$ & 1 & 1 & 0 \\
0 & 1 & 1 & 1 \\
\hline
\end{tabular}

gives us a strongly adequate matricial semantics for $P^{1}$.

The axioms of $I^{1}$ are given by the following schemas:

(A1) $A \rightarrow(B \rightarrow A)$

(A2) $(A \rightarrow(B \rightarrow C)) \rightarrow((A \rightarrow B) \rightarrow(A \rightarrow C))$

(A3') $(\neg \neg A \rightarrow \neg B) \rightarrow((\neg \neg A \rightarrow B) \rightarrow \neg A)$

$\left(\mathrm{A} 4^{\prime}\right) \neg \neg(A \rightarrow B) \rightarrow(A \rightarrow B)$

Inference rule: modus ponens [21].

$I^{1}$ is complete relative to the matrix $\mathfrak{M}^{I 1}=\left\langle\{1,1 / 2,0\}, \neg I 1, \rightarrow_{I 1},\{1\}\right\rangle$, where $\neg_{I 1}$ and $\rightarrow_{I 1}$ are defined by the tables

\begin{tabular}{|c|c|}
\hline$x$ & $\neg I 1 x$ \\
\hline 1 & 0 \\
$1 / 2$ & 0 \\
0 & 1 \\
\hline
\end{tabular}

\begin{tabular}{|c|ccc|}
\hline$\rightarrow_{I 1}$ & 1 & $1 / 2$ & 0 \\
\hline 1 & 1 & 0 & 0 \\
$1 / 2$ & 1 & 1 & 1 \\
0 & 1 & 1 & 1 \\
\hline
\end{tabular}

For ease of comparison, let's also give the axiomatization of classical propositional logic in a language using negation and implication as a primitives:

$$
\begin{aligned}
& \text { (A1) } A \rightarrow(B \rightarrow A) \\
& \text { (A2) }(A \rightarrow(B \rightarrow C)) \rightarrow((A \rightarrow B) \rightarrow(A \rightarrow C)) \\
& \text { (A3”) }(\neg B \rightarrow \neg A) \rightarrow((\neg B \rightarrow A) \rightarrow B)
\end{aligned}
$$

Inference rule: modus ponens [14, p. 35].

Let us consider paraconsistent matrix $\mathfrak{M}_{8}$ :

$\mathfrak{M}_{8}=\left\langle\{0,1 / 3,2 / 3,1\}, \neg 1, \rightarrow_{3},\{1,2 / 3\}\right\rangle$. 
The matrix $\mathfrak{M}_{8}$ can be regarded as four-valued generalizations of the three-valued matrix, introdused by Sette in [19].

The matrix $\mathfrak{M}_{8}$ generates the same theory as the well-known Sette's paraconsistent logic $P^{1}$. It follows from the paper [5, pp. 86-87], where the four-valued matrix $\mathcal{P}^{1 f}$ for logic $P^{1}$ is constructed. Matrix $\mathcal{P}^{1 \mathbf{f f}}$ is $\mathfrak{M}_{8}$ in our notation. It is shown that matrix $\mathcal{P}^{1 \mathbf{f}}$ is a homomorphic image of the matrix $\mathfrak{M}^{P 1}$ with respect to the mapping $h: h(1 / 3)=0$ and $h(x)=x$, if $x \neq 1 / 3$. As a consequence, matrix $\mathcal{P}^{1 \mathbf{f}}\left(\mathfrak{M}_{8}\right)$ induces the logic $P^{1}$ (i.e. $\mathcal{P}^{1 \mathbf{f f}}\left(\mathfrak{M}_{8}\right)$ is a characteristic matrix ${ }^{4}$ for calculus $\left.P^{1}\right)$. Moreover,

Proposition 4.1. All paraconsistent matrices, included in the upper semilattice in Figure 1 are characteristic for $P^{1}$.

Proof: The proof follows from the facts:

(1) if $\mathfrak{M}$ is a homomorphic image of $\mathfrak{N}$ then $E(\mathfrak{M})=E(\mathfrak{N})$ [3, p. 21].

(2) matrix $\mathfrak{M}_{5}$ is a homomorphic image of the matrix $\mathfrak{M}^{P 1}$ with respect to the mapping $h: h(1 / 3)=2 / 3$ and $h(x)=x$, if $x \neq 1 / 3$.

(3) matrix $\mathfrak{M}_{6}$ is a homomorphic image of the matrix $\mathfrak{M}^{P 1}$ with respect to the mapping $h: h(2 / 3)=1$ and $h(x)=x$, if $x \neq 2 / 3$.

(4) matrix $\mathfrak{M}_{7}$ is a homomorphic image of the matrix $\mathfrak{M}^{P 1}$ with respect to the mapping $h: h(1 / 3)=1$ and $h(x)=x$, if $x \neq 1 / 3$.

(5) matrix $\mathfrak{M}_{9}$ is a homomorphic image of the matrix $\mathfrak{M}^{P 1}$ with respect to the mapping $h: h(2 / 3)=0$ and $h(x)=x$, if $x \neq 2 / 3$.

Let us consider the paracomplete matrix $\mathfrak{M}_{13}$ :

$\mathfrak{M}_{13}=\left\langle\{0,1 / 3,2 / 3,1\}, \neg_{2}, \rightarrow_{3},\{1,2 / 3\}\right\rangle$.

The matrix $\mathfrak{M}_{13}$ can be regarded as four-valued generalization of threevalued matrix, introdused by Sette and Carnielli in [21].

In paper [5, p. 87] L. Devyatkin construct the four-valued matrix $\mathcal{I}^{1 \mathbf{t}}$, which is a homomorphic image of the matrix $\mathfrak{M}^{I 1}$ with respect to the mapping $h: h(2 / 3)=1$ and $h(x)=x$, if $x \neq 2 / 3$. The matrix $\mathcal{I}^{1 \mathbf{t}}$ is $\mathfrak{M}_{13}$ in our notation. It follows that the matrix $\mathfrak{M}_{13}$ generates the same theory as the paracomplete logic $I^{1}$.

\footnotetext{
${ }^{4}$ Matrix $\mathfrak{M}$ is characteristic for calculus $L$, if $\vDash_{\mathfrak{M}} A$ iff $\vdash_{L} A$.
} 
The following proposition takes place:

Proposition 4.2. All paracomplete matrices, included in the upper semilattice in Figure 1 are characteristic for $I^{1}$.

Proof: The proof follows from the facts:

(1) if $\mathfrak{M}$ is a homomorphic image of $\mathfrak{N}$ then $E(\mathfrak{M})=E(\mathfrak{N})$ [3, p. 21].

(2) matrix $\mathfrak{M}_{10}$ is a homomorphic image of the matrix $\mathfrak{M}^{I 1}$ with respect to the mapping $h: h(2 / 3)=1 / 3$ and $h(x)=x$, if $x \neq 2 / 3$.

(3) matrix $\mathfrak{M}_{11}$ is a homomorphic image of the matrix $\mathfrak{M}^{I 1}$ with respect to the mapping $h: h(1 / 3)=0$ and $h(x)=x$, if $x \neq 1 / 3$.

(4) matrix $\mathfrak{M}_{12}$ is a homomorphic image of the matrix $\mathfrak{M}^{I 1}$ with respect to the mapping $h: h(2 / 3)=0$ and $h(x)=x$, if $x \neq 2 / 3$.

(5) matrix $\mathfrak{M}_{14}$ is a homomorphic image of the matrix $\mathfrak{M}^{I 1}$ with respect to the mapping $h: h(1 / 3)=1$ and $h(x)=x$, if $x \neq 1 / 3$.

The question about the classes of tautologies generated by the matrices $\mathfrak{M}_{15}$ and $\mathfrak{M}_{16}$ is considered in paper [22]. It is proved that the theories generated by these matrices are equivalent.

The analysis of the application of the algorithm for constructing classes of literal LPP logics by combination of isomorphs of classical logic $C P C$ to three-valued and four-valued cases allows us to make two more general assumptions:

1. All paraconsistent (and not paracomplete) matrices constructed by combination of isomorphs of classical logic $C P C$ generate the same theory as Sette's paraconsistent logic $P^{1}[19]$.

2. All paracomplete (and not paraconsistent) matrices constructed by combination of isomorphs of classical logic $C P C$ generate the same theory as paracomplete logic $I^{1}$ introdused by Sette and Carnielli in [21].

\subsection{Some properties of lattices}

For our analysis, we have chosen the lattices, presented in Figures 2 and 3, because it helps us to demonstrate, how it is possible to vary paraconsistency and paracompleteness properties in logics. 
Let us consider the lattice in Figure 2. Martices $\mathfrak{M}_{8}, \mathfrak{M}_{13}, \mathfrak{M}_{15}$, corresponding to LPP logics, differ only in negation operation. It's obvious that negation $\neg_{1}$ is paraconsistent in the sense that classical negation allows explosity, but the negation $\neg_{1}$ does not, that is $A$ and $\neg_{1} A$ can be true at the same time. Negation $\neg_{2}$ has the property of paracompleteness, in the sense that $A$ and $\neg_{2} A$ can be false at the same time. Thus, by varying the negation operation, we can obtain LPP logics with different properties. And herein the implication operation and the class of designated values in the matrices remain the same. Taking this into account, it is clearly seen that it is natural to axiomatize the LPP logics, varying the axioms for negation. We see this on the example of the foregoing axiomatizations of $P^{1}, I^{1}$ and $C P C$. The matrices $\mathfrak{M}_{8}$ and $\mathfrak{M}_{13}$ are four-valued characteristic matrices for the known calculi $P^{1}$ and $I^{1}$, and differ only in the negation operation.

Let us turn to the lattice in Figure 3. Here, the matrices of the corresponding LPP logics differ in the implication operations and in the class of the designated values. Herein the negation operation is defined by same truth-table in all these matrices $\left(\mathfrak{M}_{3}, \mathfrak{M}_{6}, \mathfrak{M}_{11}, \mathfrak{M}_{16}\right)$ and properties of negation operation (and corresponding LPP logics) are directly dependent on the choice of the designated values class.

The paraconsistent logic corresponding to the matrix $\mathfrak{M}_{6}$ in Lattice 1 is functionally eqiuvalent to the paracomplete logic corresponding to the matrix $\mathfrak{M}_{13}$ in Lattice 2; and the paraconsistent logic corresponding to the matrix $\mathfrak{M}_{11}$ in Lattice 2 is functionally eqiuvalent to the paracomplete logic corresponding to the matrix $\mathfrak{M}_{8}$ in Lattice 1 . Both paraconsistent logics generate the same theory as well-known Sette's paraconsistent logic [19], and paracomplete logics have the same set of tautologies as the paracomplete logic $I^{1}[21]$.

\section{Concluding remarks}

We have analized the application of the method of constructing LPP logics by combinating isomorphs of classical logic to the four-valued case. Recall that in the case of three-valued logics Sette's paraconsistent logic $P^{1}$ and paracomplete logic $I^{1}$ can be obtained by using this method. As a result we get four-valued generalizations of these logics. 
This method preserves all essential properties of these LPP logics, i.e. allows to construct paraconsistent and paracomplete matrices, which are functionally equivalent, on the one hand, and generate theories equivalent to $P^{1}$ and $I^{1}$, on the other. In the three-valued case, combination of isomorphs of $C P C$ leads to two LPP logics, in four-valued case, we can obtain $^{5}$ four isomorphs of $C P C$, combinating which allows to obtain five paraconsistent, five paracomplete and two paranormal logics. At that, these LPP logics form the 10-element upper semi-lattice with respect to the functional embeddinig one logic into another.

And the foregoing upper semi-lattice includes several four-element lattices with respect to the functional embeddinig one logic into another, on the one hand, and with respect to the possesion of paraconsistency and paracompleteness properties, on the other. Two such four-element lattices of LPP logics were considered. Functional properties and sets of tautologies of corresponding LPP logics were investigated. On the example of these two lattices the mechanism of variation of paraconsistency and paracompleteness properties in logics is clearly seen.

As a result, the analysis allows us to make an assumption that all $n$-valued literal paraconsistent matrices (and not paracomplete) and all $n$-valued literal paracomplete matrices (and not paraconsistent) constructed by combinating isomorphs of classical logic generate the same theories as $P^{1}$ and $I^{1}$.

\section{References}

[1] O. Arieli, A. Avron, Four-valued paradefinite logics, Studia Logica, vol. 105(6) (2017), pp. 1087-1122, DOI: https://doi.org/10.1007/s11225-0179721-4.

[2] D. A. Bochvar, V. K. Finn, On many-valued logics admitting formalization of the analysis of antinomies. 1, [in:] Studies in mathematical linguistics, mathematical logic and information languages, Nauka, Moscow (1972), pp. 238-295.

[3] L. Bolc, P. Borowik, Many-valued Logics: 1: Theoretical Foundations, Springer-Verlag Berlin Heidelberg (1992).

\footnotetext{
${ }^{5}$ By the method specified in section 3.
} 
[4] J. Ciuciura, A weakly-intuitionistic logic I1, Logical Investigations, vol. 21(2) (2015), pp. 53-60.

[5] L. Y. Devyatkin, On a continual class of four-valued maximally paranormal logics, Logical Investigations, vol. 24(2) (2018), pp. 85-91, DOI: https: //doi.org/10.21146/2074-1472-2018-24-2-85-91, in Russian.

[6] V. L. Fernández, Semântica de Sociedades para Lógicas $n$-valentes, Master's thesis, Campinas: IFCH-UNICAMP (2001).

[7] V. L. Fernández, M. E. Coniglio, Combining valuations with society semantics, Journal of Applied Non-Classical Logics, vol. 13(1) (2003), pp. 21-46, DOI: https://doi.org/10.3166/jancl.13.21-46.

[8] S. Jaśkowski, A propositional calculus for inconsistent deductive systems, Studia Logica, vol. 24 (1969), pp. 143-157.

[9] A. Karpenko, N. Tomova, Bochvar's three-valued logic and literal paralogics: the lattice and functional equivalence, Logic and Logical Philosophy, vol. 26(2) (2017), pp. 207-235, DOI: https://doi.org/10.12775/LLP.2016. 029 .

[10] A. S. Karpenko, Jaśkowski's criterion and three-valued paraconsistent logics, Logic and Logical Philosophy, vol. 7 (1999), pp. 81-86, DOI: https: //doi.org/10.12775/LLP.1999.006.

[11] A. S. Karpenko, A maximal paraconsistent logic: The combination of two three-valued isomorphs of classical propositional logic, [in:] D. Batens, C. Mortensen, G. Priest, J.-P. Van Bendegem (eds.), Frontiers of Paraconsistent Logic, Baldock Research Studies Press (2000), pp. 181-187.

[12] A. S. Karpenko, N. E. Tomova, Bochvar's three-valued logic and literal paralogics, Institute of Philosophy of Russian Academy of Science, Moscow (2016).

[13] R. A. Lewin, I. F. Mikenberg, Literal-paraconsistent and literal-paracomplete matrices, Mathematical Logic Quarterly, vol. 52(5) (2006), pp. 478-493, DOI: https://doi.org/10.1002/malq.200510044.

[14] E. Mendelson, Introduction to Mathematical Logic, 4th ed., Chapman \& Hall (1997).

[15] Y. I. Petrukhin, Deduction Normalization Theorem for Sette's Logic and Its Modifications, Moscow University Mathematics Bulletin, vol. 74(1) (2019), pp. 25-31, DOI: https://doi.org/10.3103/S0027132219010054. 
[16] V. M. Popov, On the logics related to A. Arruda's system V1, Logic and Logical Philosophy, vol. 7 (1999), pp. 87-90, DOI: https://doi.org/10. 12775/LLP.1999.007.

[17] G. Priest, K. Tanaka, Z. Weber, Paraconsistent logic (2013), URL: http: //plato.stanford.edu/entries/logic-paraconsistent, Stanford Encyclopedia of Philosophy.

[18] L. Z. Puga, N. C. A. Da Costa, On the imaginary logic of N. A. Vasiliev, Zeitschrift für mathematische Logik und Grundlagen der Mathematik, vol. 34 (1988), pp. 205-211.

[19] A. M. Sette, On propositional calculus P1, Mathematica Japonicae, vol. 18 (1973), pp. 173-180.

[20] A. M. Sette, E. H. Alves, On the equivalence between some systems of non-classical logic, Bulletin of the Section of Logic, vol. 25(2) (1973), pp. 68-72.

[21] A. M. Sette, W. A. Carnielli, Maximal weakly-intuitionistic logics, Studia Logica, vol. 55(1) (1995), pp. 181-203, DOI: https://doi.org/10.1007/ BF01053037.

[22] N. E. Tomova, On properties of a class of four-valued papranormal logics, Logical Investigations, vol. 24(1) (2018), pp. 75-89, DOI: https://doi. org/10.21146/2074-1472-2018-24-1-75-89.

[23] N. E. Tomova, A. N. Nepeivoda, Functional properties of four-valued paralogics, Logical-Philosophical Studies, vol. 16(1-2) (2018), pp. 130-132.

\section{Natalya Tomova}

Russian Academy of Sciences

Institute of Philosophy

Goncharnaya 12/1

109240 Moscow, Russian Federation

e-mail: natalya-tomova@yandex.ru 